\title{
The 'step-clipping' method: a guide for identifying a previously bleeding colonic diverticulum $\square$
}

\section{다 (1) $\odot$}

\author{
Authors \\ Institution \\ Department of Gastroenterology, Hiroshima City Asa \\ Citizens Hospital, Hiroshima, Japan \\ submitted 4.6.2019 \\ accepted after revision 12.9.2019 \\ Bibliography \\ DOI https://doi.org/10.1055/a-1027-6830 | \\ Endoscopy International Open 2020; 08: E64-E69 \\ (c) Georg Thieme Verlag KG Stuttgart · New York \\ elSSN 2196-9736 \\ Corresponding author \\ Taiki Aoyama, Department of Gastroenterology, Hiroshima \\ City Asa Citizens Hospital, 2-1-1 Kabe-minami, Asakita-ku, \\ Hiroshima 731-0293, Japan \\ Fax: +81-82-814-1791 \\ t-aoyama@asa-hosp.city.hiroshima.jp
}

Hirosato Tamari, Taiki Aoyama, Kenjiro Shigita, Naoki Asayama, Akira Fukumoto, Shinichi Mukai, Shinji Nagata

\section{ABSTRACT}

Background and study aims Unsatisfactory detectability of a previously bleeding diverticulum by colonoscopy results from difficulty in precisely locating the target lesion, even with presence of an extravasation on contrast-enhanced computed tomography (CECT). This study aimed to evaluate the usefulness of the step-clipping method to overcome this limitation.

Patients and methods Step-clipping was indicated for patients with colonic diverticular bleeding and presence of extravasation on CECT, but with absence of active bleeding on subsequent colonoscopy. The target diverticulum was identified by comparing computed tomography images before and after step clipping, which provided a positional relationship between each clip and the target lesion.

Results Based on data from 21 consecutive cases meeting our inclusion criteria (14 men and 7 women; mean age, 73.2 years), the target diverticulum was endoscopically identified in 20 cases (95\%), in a median time of 5 minutes, and successfully treated. No adverse events were observed with the step-clipping method.

Conclusion Step-clipping provided easy guidance to the target site for treatment in a short time, despite spontaneous cessation of bleeding at the diverticulum.

\section{Introduction}

Depiction of extravasation on contrast-enhanced computed tomography (CECT) is definitive evidence of colonic diverticular bleeding and provides endoscopists with information on the region of interest for colonoscopic investigation. Identification of an extravasation on CECT increases the detection rate of a bleeding diverticulum by colonoscopy to $50 \%$ to $68.3 \%$, compared to $20.2 \%$ to $36.3 \%$ in the absence of an observable extravasation [1,2]. However, even with positive CECT information, the detection rate of a bleeding diverticulum remains unsatisfactory when spontaneous cessation of bleeding occurs. Moreover, as colonoscopy does not provide precise positional information, compared to computed tomography (CT), detectability of the target diverticulum during colonoscopy depends on the skill of the endoscopist and on chance.
The "step-clipping" method overcomes this limitation by making artificial signposts in the colon using endoclips, thus providing the positional relationship between the clips and the target diverticulum responsible for the bleeding [3]. In this way, the endoscopist can navigate to the target site by referring to the positional information from each clip, rather than performing a time-consuming search in cases of spontaneous cessation of bleeding at the target diverticulum. Accordingly, the aim of our study was to evaluate the usefulness of the step-clipping method for endoscopic navigation to a target diverticulum.

\section{Patients and methods}

\section{Patients}

Prospective patients were those presenting with a chief complaint of hematochezia at our hospital, between June 2017 and January 2019. Of them, those with evidence of extravasation on 

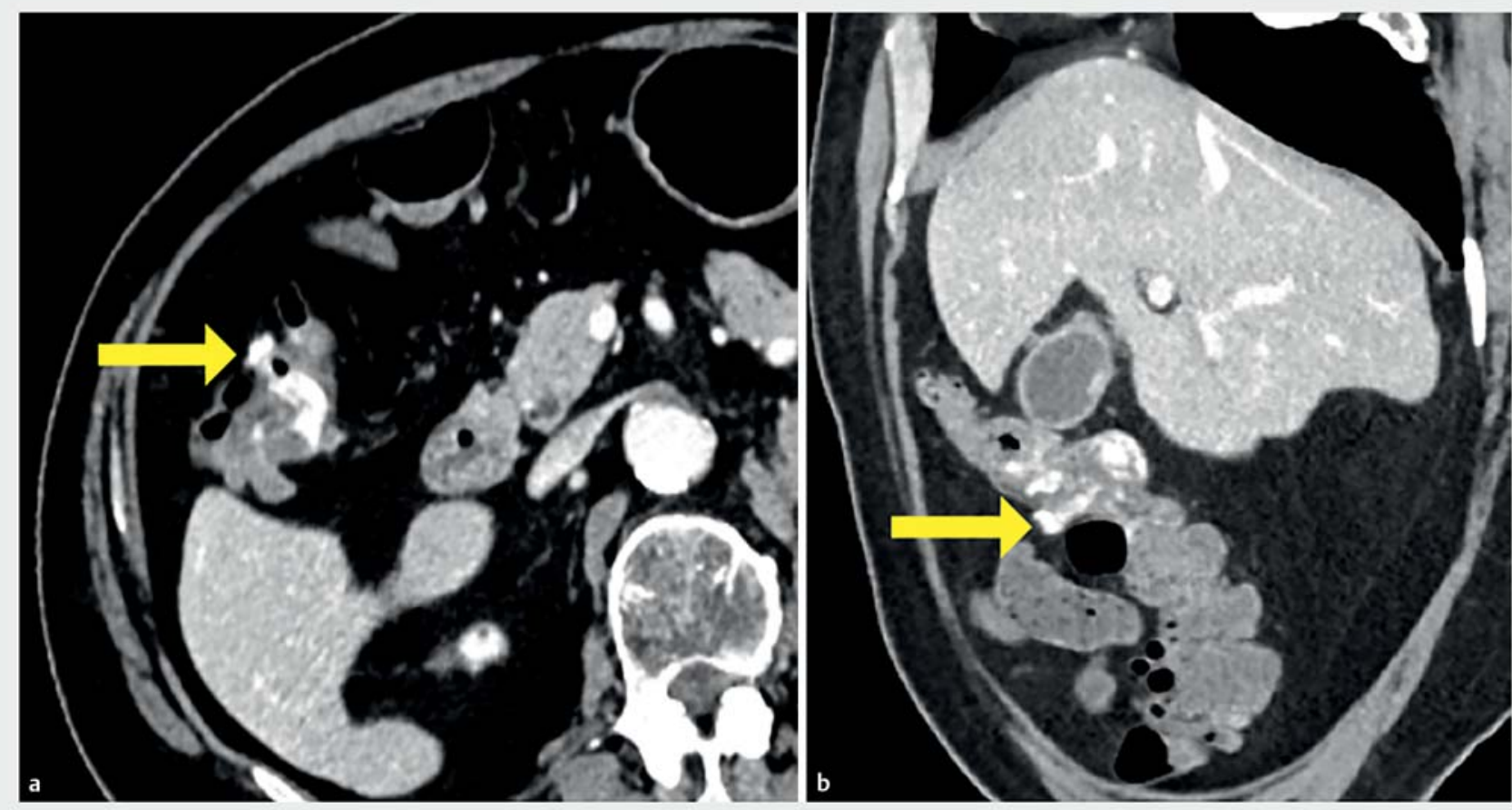

Fig. 1 Extravasation observed on contrast-enhanced computed tomography images obtained on the day of admission. a Axial image showing the bleeding (target) lesion in the ascending colon, among numerous diverticula (yellow arrow). b Sagittal image showing the target lesion located in the middle of the ascending colon (yellow arrow).

CECT, but with absence of active bleeding on subsequent colonoscopy, were candidates for the step-clipping method. Patients for whom bowel preparation or colonoscopy examination were contraindicated due to extreme age or multiple comorbidities were excluded. We also excluded patients for whom CECT was contraindicated due to renal malfunction or allergy to the enhancement agent.

\section{Step-clipping method}

CT imaging was performed using a 64-slice diagnostic imaging technique on a Somatom Definition Flash system (Siemens Healthineers, Erlangen, Germany). For patients with an extravasation detected on CECT ( $>$ Fig. 1), an initial colonoscopy was performed using a PCF-Q260AZI endoscope (Olympus, Tokyo, Japan), under $\mathrm{CO}_{2}$ insufflation, after antegrade bowel preparation using 2 liters of polyethylene glycol (PEG). When a site of active bleeding was not identified, several clips were placed, 2 inches apart, including at the site of observed extravasation on CECT images. The range and number of clips deployed was dependent on the certainty of the location, which was determined in reference to the presumptive location of the cecum, hepatic flexure, splenic flexure, and junction of the sigmoid-descending colon on transluminal viewing colonoscopy, with this wide region certainly including the specific region of interest. The placement of endoclips was performed with minimal search in order not to change the environment of the colon ( $>$ Fig.2). After placement, the scope was promptly removed and unenhanced CT imaging was conducted 2 hours later. On the unen- hanced CT image, the positional relationship between each clip and the target diverticulum, identified on the initial CECT, was evaluated using 1-mm axial image slices, with coronal or sagittal plane images added as needed ( $\triangleright$ Fig. 3 ). The target diverticulum was tracked and identified on the $\mathrm{CT}$ images in reference to the neighboring symbolic structures (e.g., fecal-impacted diverticulum and large-sized diverticulum). CT images were reviewed and interpreted by two endoscopists, each of whom had previously performed more than 1000 colonoscopies, with a conclusive diagnosis reached by agreement. Based on the evaluation, secondary colonoscopy was conducted to identify the target diverticulum ( $\triangleright$ Fig.4). All endoscopic procedures were conducted during the day, regardless of the time of admission. No fluoroscopy was used.

\section{Statement of ethics}

This study conformed to the principles of the $6^{\text {th }}$ revision of the Declaration of Helsinki (2008), and the study protocol was approved by the institutional review board (IRB) of the Hiroshima City Asa Citizens Hospital, which also granted us permission to access the patients' information on April 19, 2019 (IRB No.311-10). Owing to the retrospective design, the need for patient consent was waived. 


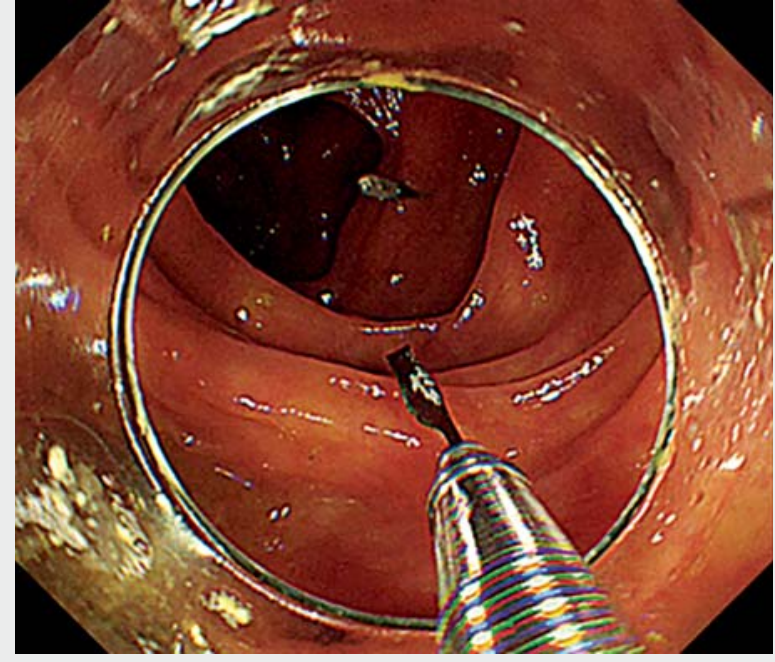

a<smiles>[Te]</smiles>

Y

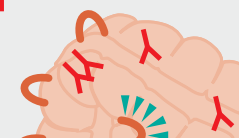

$\lambda$

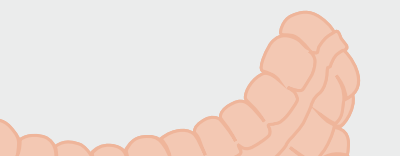

b

- Fig. 2 The step-clipping method performed at initial colonoscopy. a Marking clips, placed 2 inches apart, including the suspected site. $\mathbf{b}$ The placement scheme of the marking clips.

\section{Results}

Over the defined study period, 89 patients were admitted to our hospital with a diagnosis of colonic diverticular bleeding. Extravasation on CECT was observed in 25 of these patients. Of them, four were excluded for the following reasons: contraindication to colonoscopy due to extreme age $(n=2)$ and active bleeding during initial colonoscopy $(n=2)$. Therefore, our analysis included data from 21 consecutive patients ( 14 men and 7 women; mean age, 73.2 years). Ten patients (48\%) presented with severe bleeding, based on the need for red blood cell transfusion or a decrease in hematocrit $\geq 20 \%$ [4]. The average (standard deviation) severity of bleeding score was 3.1 [1.2], according to the NOBLADS system [5]. Extravasation was loca-
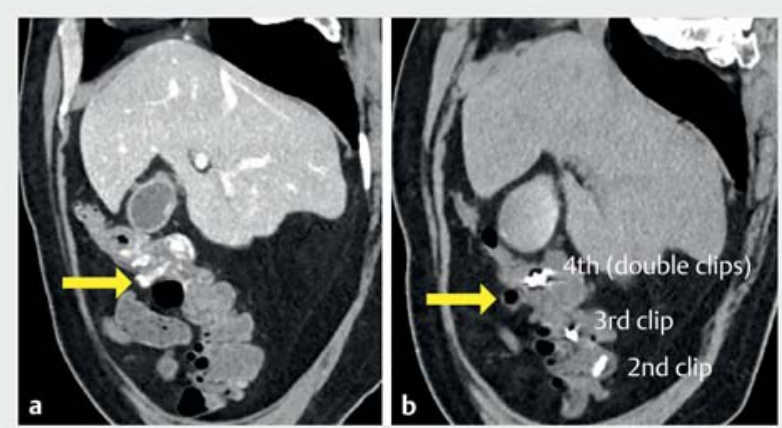

- Fig. 3 Comparison of the sagittal computed tomography images before and after step-clipping. a Contrast-enhanced image obtained on the day of admission (before step-clipping). The target lesion is located in the middle of the ascending colon (yellow arrow). b Unenhanced computed tomographic image obtained after step-clipping, with the target diverticulum identified opposite to the fourth clip (yellow arrow).

ted in the ascending colon in nine cases, transverse colon in four, descending colon in three, and sigmoid colon in five. Fifteen cases (71\%) had clustering diverticula, with a density of $>10$ per 2 inches, in the colon. Relevant characteristics of the study group are summarized in $>$ Table $\mathbf{1}$.

The target diverticulum was identified in 20 of 21 cases (95\%; - Table 2). The target diverticulum was not identified in the ascending colon in one case after 58 minutes of endoscopic examination. In that patient, numerous diverticula were identified in the ascending colon, but none of them were identified as a potential source of bleeding. Median endoscopic search time to identify the target diverticulum was 5 minutes (range, 1 to 58 minutes), with a median total examination time of 40 minutes (range, 20 to 85 minutes), which included insertion of the endoscope, step-clipping, searching, and treatment, combined for both the initial and secondary colonoscopy. In all cases, no active bleeding was identified during the colonoscopy, with the target diverticulum confirmed by evidence of clot attachment in nine cases and non-bleeding visible vessels in the other 11 cases. Endoscopic band ligation (EBL) was used as the primary treatment in all cases, with one case requiring subsequent clipping and another case requiring arterial embolization. Median time of total work-up from admission to the second colonoscopy was 32 hours (range, 14 to 136 hours). There was no incidence of adverse events, with no report of rebleeding after treatment, over a 3-month period of follow-up.

\section{Discussion}

Colonic diverticulosis is a common disease that is frequently observed during colonoscopy. The etiology of colonic diverticulosis has not been fully clarified, although aging is considered as a definite risk factor [6]. Among patients with colonic diverticula, $15 \%$ to $30 \%$ experience some diverticula-related event during their lifetime, such as inflammation or bleeding [7]. Extravasation on CECT is definitive evidence of colonic diverticular 

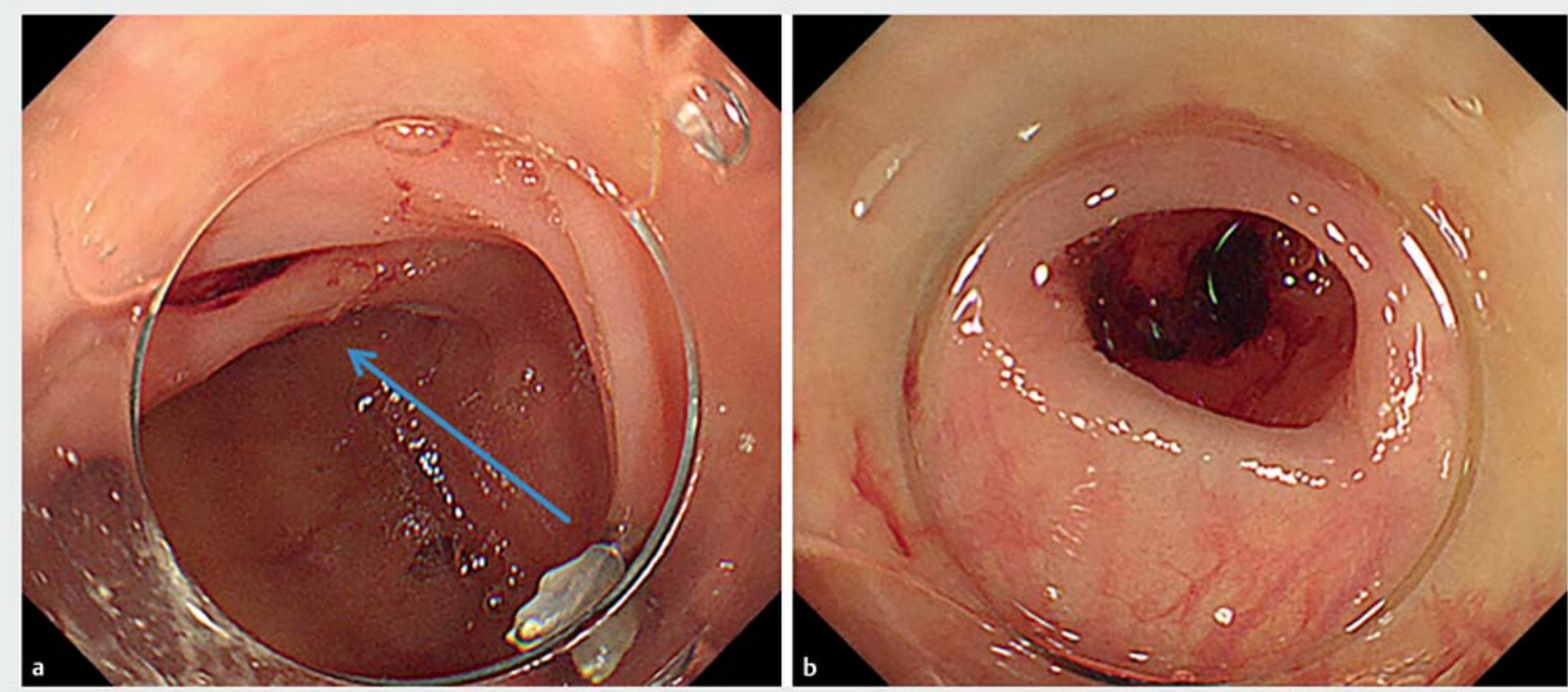

- Fig. 4 Identification of the target lesion on secondary colonoscopy. a Based on evaluation of computed tomography images, the colonoscope was immediately navigated to the suspicious site, located opposite to the fourth clip (blue arrow). $\mathbf{b}$ The target lesion was successfully identified.

bleeding and thus, helps in planning of the colonoscopy examination. However, because of the intermittent nature of colonic diverticular bleeding, the detection rate of the target lesion by colonoscopy remains at $\leq 70 \%[1,2]$. Moreover, colonoscopy does not provide positional information about the target diverticulum that is as precise as CT or barium enema [8]. Because of the low detection rate of the target diverticulum, endoscopic examination can typically be extended to 45 to 140 minutes, with the examination becoming troublesome [9-11]. Step-clipping has been advocated as a breakthrough technique for the management of a diverticulum suspected of causing bleeding, detected by extravasation on CECT, but with absence of active bleeding on subsequent colonoscopy. When active bleeding is present, endoscopic hemostasis can be directly applied. In the absence of active bleeding, step-clipping can facilitate identification of the target diverticulum, especially in the presence of other residual clots in the colon. In our study, the step-clipping technique was associated with a $95 \%$ detection rate, with a short examination (mean, 11.2 minutes) needed to identify the target lesion.

As the step-clipping technique is based on an analysis of CT images, the method affords endoscopists the time needed to examine the colon, free of the stress associated with extending an endoscopic examination. Even in the presence of intestinal peristalsis or pooled gas on the CT image, these features do not have significant effects on the primary colon structure or on the interpretation of the image. Therefore, CT-based preplanning eliminates the need for redundant searching for the target lesion during the second colonoscopy for treatment, even when active bleeding is not present. In our protocol, unenhanced CT was performed 2 hours after step-clipping, which provided sufficient time for $\mathrm{CO}_{2}$ to be either completely absor- bed or excreted. This step is important to ensure that the colon is deflated, allowing comparison of the before and after $\mathrm{CT}$ images. In our study, we used 1-mm-axial before and after images, with the 1-mm thick slices providing a more appropriate method to identify a probable bleeding diverticulum than 5-mm CT slices, particularly for small-size diverticula.

A previous study reported on the effectiveness of colonoscopy performed within 24 hours from the onset of hematochezia [12]. By comparison, step-clipping can be conducted regardless of the time to endoscopy from the onset of hematochezia, in a well-prepared environment with PEG bowel preparation. Although it is true that early colonoscopy may improve outcomes in patients with acute lower gastrointestinal bleeding, urgent CT before colonoscopy provided $15 \%$ additional detectability compared to colonoscopy alone [13]. The step-clipping method achieves a high detection rate of the target diverticulum, even in cases requiring elective colonoscopy. Therefore, with accessibility to $\mathrm{CT}$ imaging, the step-clipping method provides a clinically feasible method to identify the target diverticulum for treatment, which could be implemented in clinics with insufficient human or material resources to conduct urgent colonoscopy.

The step-clipping method has two primary limitations that should be acknowledged. First, step-clipping requires additional costs (about \$270) and CT examination (an effective dose of radiation of about $5 \mathrm{mGy}$ ), compared to the traditional approach combining CECT and colonoscopy. Although this is troublesome, consistent detection and treatment of the target lesion might reduce the rate of rebleeding and readmission, lowering costs and radiation exposure in the longer term. Further investigation of the clinical impact of the step-clipping method, through the accumulation of experience, is warranted. Sec- 
- Table 1 Characteristics of the patient group.

\begin{tabular}{|c|c|}
\hline Variable & $N=21$ \\
\hline Age, mean [SD], years & $73.2[12.8]$ \\
\hline Sex (male/female), n & $14 / 7$ \\
\hline \multicolumn{2}{|l|}{ Severity of bleeding } \\
\hline - Hemoglobin level on admission, mean [SD], g/dL & $11.9[2.1]$ \\
\hline - Red blood cell transfusion needs, n (\%) & $9(43)$ \\
\hline - Decrease in hematocrit $\geq 20 \%, n(\%)$ & $4(19)$ \\
\hline - ${ }^{1}$ NOBLADS score, mean [SD] & $3.1[1.2]$ \\
\hline \multicolumn{2}{|l|}{ Distribution of diverticula, n (\%) } \\
\hline - Right-side colon & $17(81)$ \\
\hline - Left-side colon & $18(86)$ \\
\hline \multicolumn{2}{|l|}{ Location of the extravasation on CECT, n (\%) } \\
\hline - Ascending colon & $9(43)$ \\
\hline - Transverse colon & $4(19)$ \\
\hline - Descending colon & $3(14)$ \\
\hline - Sigmoid colon & $5(24)$ \\
\hline \multicolumn{2}{|l|}{ Density of diverticula (per 2 inches), $n$} \\
\hline$\cdot>10$ & 15 \\
\hline - $\geq 5$ and $\leq 10$ & 4 \\
\hline.$<5$ & 2 \\
\hline \multicolumn{2}{|c|}{$\begin{array}{l}\text { SD, standard deviation; CECT, contrast-enhanced computed tomography } \\
1 \text { Scoring system based on nonsteroidal anti-inflammatory drugs use, no } \\
\text { diarrhea, no abdominal tenderness, blood pressure of } 100 \mathrm{mmH} \text { or lower, } \\
\text { antiplatelet drugs use, albumin level less than } 3.0 \mathrm{~g} / \mathrm{dL} \text {, disease score of } 2 \\
\text { or higher, and syncope. }\end{array}$} \\
\hline
\end{tabular}

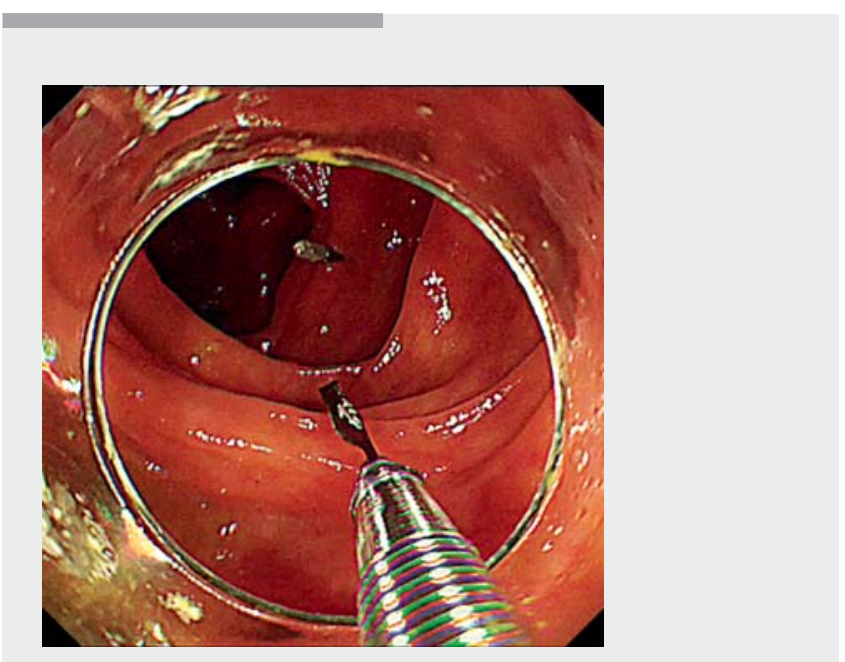

$\checkmark$ Video 1 Placement of the marking clips and subsequent computed tomography provide objective positional information, which can lead to rapid and precise identification of the responsible diverticulum on subsequent colonoscopy.
Table 2 Endoscopic management.

\begin{tabular}{|l|c|}
\hline Variable & $\mathbf{N}=\mathbf{2 1}$ \\
\hline Number of deployed clips, median (range) & $8(3-12)$ \\
\hline Detection of target diverticulum, $\mathrm{n}(\%)$ & $20(95)$ \\
\hline Total examination time, minutes & \\
\hline - Mean [SD] & $41.5[20.1]$ \\
\hline - Median (range) & $40(20-85)$ \\
\hline Total searching time, minutes & \\
\hline - Mean [SD] & $11.2[16.9]$ \\
\hline - Median (range) & $5(1-58)$ \\
\hline Type of SRH, $\mathrm{n}(\%)$ & \\
\hline - Clot attachment & $9(43)$ \\
\hline - Non-bleeding visible vessel & $11(52)$ \\
\hline Treatment, $\mathrm{n}(\%)$ & $18(86)$ \\
\hline - EBL & $1(5)$ \\
\hline - EBL+ clipping & $1(5)$ \\
\hline - EBL+ clipping + arterial embolization & $0(0)$ \\
\hline - Adverse event, $\mathrm{n}$ (\%) & \\
\hline
\end{tabular}

SD, standard deviation; SRH, stigmata of recent hemorrhage; EBL, endoscopic band ligation.

ond, the step-clipping method can only be used in cases where extravasation is identified on CECT.

\section{Conclusions}

Based on our analysis, we believe that step-clipping provides an effective map to facilitate navigation to the target diverticulum within a short time, even in the absence of active bleeding, during endoscopy.

Competing interests

None

References

[1] Nakatsu S, Yasuda H, Maehata T et al. Urgent computed tomography for determining the optimal timing of colonoscopy in patients with acute lower gastrointestinal bleeding. Intern Med 2015; 54: 553-558

[2] Obana T, Fujita N, Sugita R et al. Prospective evaluation of contrastenhanced computed tomography for the detection of colonic diverticular bleeding. Dig Dis Sci 2013; 58: 1985-1990

[3] Tamari H, Aoyama T, Nagata S et al. "Step-clipping" method: a technique for detecting previously bleeding diverticula. Endoscopy 2018; 50: E10-E12 
[4] Strate LL, Orav EJ, Syngal S. Early predictors of severity in acute lower intestinal tract bleeding. Arch Intern Med 2003; 163: 838-843

[5] Aoki T, Nagata N, Shimbo T et al. Development and validation of a risk scoring system for severe acute lower gastrointestinal bleeding. Clin Gastroenterol Hepatol 2016; 14: 1562-1570

[6] Peery AF, Sandler RS, Ahnen DJ et al. Constipation and a low-fiber diet are not associated with diverticulosis. Clin Gastroenterol Hepatol 2009; 11: 1622-1627

[7] Stollman N, Raskin JB. Diverticular disease of the colon. Lancet 2004; 363: 631-639

[8] Niikura R, Nagata N, Shimbo T et al. Colonoscopy can miss diverticula of the left colon identified by barium enema. World J Gastroenterol 2013; 19: 2362-2367

[9] Shimamura Y, Ishii N, Omata F et al. Endoscopic band ligation for colonic diverticular bleeding: possibility of standardization. Endosc Int Open 2016; 4: E233-E237
[10] Yen EF, Ladabaum U, Muthusamy VR et al. Colonoscopic treatment of acute diverticular hemorrhage using endoclips. Dig Dis Sci 2008; 53 : 2480-2485

[11] Farrell J], Graeme-Cook F, Kelsey PB. Treatment of bleeding colonic diverticula by endoscopic band ligation: an in-vivo and ex-vivo pilot study. Endoscopy 2003; 35: 823-829

[12] Niikura R, Nagata N, Aoki T et al. Predictors for identification of stigmata of recent hemorrhage on colonic diverticula in lower gastrointestinal bleeding. J Clin Gastroenterol 2015; 49: e24-e30

[13] Nagata N, Niikura R, Aoki T et al. Role of urgent contrast-enhanced multidetector computed tomography for acute lower gastrointestinal bleeding in patients undergoing early colonoscopy. | Gastroenterol 2015; 50: 1162-1172 